\title{
Supreme Pfister Forms
}

\author{
Karim Johannes Becher
}

\begin{abstract}
For a nonreal field $F$ of characteristic different from 2, we compare several properties which $F$ may have with respect to an anisotropic $n$-fold Pfister form $\pi$ over $F$. In particular, we consider the situation where the subforms of $\pi$ are all the anisotropic quadratic forms over $F$; then $\pi$ is said to be supreme. We further apply our results to the case where $\pi$ is the form $2^{n} \times\langle 1\rangle(n \geq 1)$. In this way we obtain new examples of nonreal fields with prescribed level and with additional properties.
\end{abstract}

\section{Introduction}

Throughout this paper let $F$ be a field of characteristic different from 2 . We denote by $F^{\times}$the multiplicative group of $F$ and by $F^{\times^{2}}$ the subgroup of nonzero squares in $F$. Their quotient $F^{\times} / F^{\times^{2}}$ may be considered as a vector space over $\mathbb{F}_{2}$, the field with two elements. Let

$$
h: F^{\times} / F^{\times^{2}} \times F^{\times} / F^{\times 2} \longrightarrow \mathbb{F}_{2}
$$

denote the map which associates to a couple $\left(a F^{\times 2}, b F^{\times^{2}}\right)$ the value 0 if the equation $a X^{2}+b Y^{2}=1$ has a solution over $F$ and the value 1 otherwise. If $F$ is a local field then $h$ is closely related to the Hilbert symbol of $F$.

Fröhlich investigated in [6] when the field $F$ satisfies the following condition: $F$ is not quadratically closed and for any quadratic extension $K / F$ the group $N(K / F)$ of nonzero norms has index two in $F^{\times}$. This turns out to be equivalent to the condition that the map $h$ is $\mathbb{F}_{2}$-bilinear and nondegenerate. Further, in this situation $h$ determines to a large extent the quadratic form structure of the field $F$. Fröhlich calls $F$ a generalized local field if it satisfies his condition. Apart from local fields, also real closed fields fall under this definition.

Inspired by Fröhlich's observations, Kaplansky defined in [9] generalized Hilbert fields as fields of characteristic not 2 which have a unique anisotropic 
2-fold Pfister form. He shows that $F$ is a generalized Hilbert field if and only if the map $h$ is $\mathbb{F}_{2}$-bilinear and nontrivial. Kaplansky explains that one can then carry out "abstract" quadratic form theory modulo the radical $\mathrm{R}(F)$ of $F$, a subgroup of $F^{\times}$taking the place of $F^{\times^{2}}$. (If $F$ is a generalized Hilbert field then $\mathrm{R}(F)$ is such that $\mathrm{R}(F) / F^{\times 2}$ is the radical of the bilinear form $h$.)

Later, Szymiczek considered in [20] fields which have a unique anisotropic $n$-fold Pfister form, where $n$ is any positive integer. Extending an observation of Kaplansky for $n=2$, he shows that such a form exists over $F$ if and only if 2 is the maximal index in $F^{\times}$of the subgroup consisting of the elements represented by an $(n-1)$-fold Pfister form over $F$. Further, for any $n$ he gives examples of such fields with prescribed square class number, at least equal to $2^{n}$.

This article discusses other possible generalizations of the property for fields studied by Fröhlich. We introduce and compare several properties which the field $F$ may have with respect to an anisotropic Pfister form and we try to determine the consequences for the quadratic form structure of $F$. All the considered properties hold in particular for any local field (of characteristic different from 2) together with its unique anisotropic 2-fold Pfister form. In the first place we will investigate the condition that $F$ has a supreme form, i.e. an anisotropic Pfister form $\pi$ which contains every other anisotropic form over $F$ as a subform. We shall see how examples of such fields may be obtained. Apart from the aforementioned results, this work has been inspired by the author's interest in the study of levels of fields. The last section of the paper is therefore devoted to fields having as supreme form the form $2^{n} \times\langle 1\rangle$; such fields exist and they have level $2^{n}$ and some further properties.

Before giving a more detailed description of the content of the following sections, we make some conventions. By a "quadratic form" or even just a "form" we always mean a nondegenerate finite-dimensional quadratic form. We distinguish quadratic forms only up to isometry. So we may say that two forms $\varphi_{1}$ and $\varphi_{2}$ over $F$ are equal if they are isometric; we write $\varphi_{1} \cong \varphi_{2}$ in this case, however, in order to avoid confusion with Witt equivalence. In particular, by the uniqueness of a form subject to certain conditions we always mean uniqueness up to isometry. Further, we will not consider the trivial form (on the zero space) as anisotropic, thus anisotropic forms are understood to be of positive dimension.

We denote the Witt ring of $F$ by $W(F)$ and its fundamental ideal by $I(F)$. For $m \geq 0$, we use the notations $I^{m} F$ for $(I(F))^{m}$, the $m$-th power of the fundamental ideal, and $\bar{I}^{m} F$ for the quotient $I^{m} F / I^{m+1} F$. 
We define the level of $F$ as

$$
s(F):=\sup \{s \in \mathbb{N} \mid s \times\langle 1\rangle \text { is anisotropic over } F\} .
$$

This is equivalent to the usual definition of the level, that is, $s(F)$ is the least positive integer $s$ such that -1 is a sum of $s$ squares in $F$ whenever such an integer exists and $s(F)$ is equal to $\infty$ otherwise. Recall that $s(F)=\infty$ is equivalent to the existence of an ordering on $F$ and that $F$ is called a (formally) real field in this case. If $s(F)<\infty$, i.e. if -1 is a sum of squares in $F$, then the field $F$ is said to be nonreal.

Suppose now that $F$ is nonreal. For $s:=s(F)<\infty$ we call the form $s \times\langle 1\rangle$ over $F$ the level form of $F$. It follows from the crucial properties of Pfister forms that the level form of $F$ is a Pfister form, so in particular that $s$ is a power of 2 , as demonstrated by Pfister [17].

The $u$-invariant of the field $F$, here assumed to be nonreal, is defined by

$$
u(F):=\sup \{\operatorname{dim}(\varphi) \mid \varphi \text { is an anisotropic quadratic form over } F\} .
$$

Whenever finite, $u(F)$ is the smallest integer $u$ such that any form of dimension at least $u$ is universal.

In Section 3 we investigate when there exists an anisotropic form $\varphi$ over $F$ such that every anisotropic form over $F$ is a subform of $\varphi$. In our terminology this means that $\varphi$ is the supreme form of $F$. We show that a supreme form is necessarily a Pfister form (3.2). The existence of a supreme form over $F$ implies immediately that $F$ is a nonreal field of finite $u$-invariant. In (3.5) we obtain further consequences of the condition that $F$ has a supreme $n$-fold Pfister form, in particular concerning the powers of the fundamental ideal in the Witt ring of $F$. In order to show that a certain anisotropic $n$-fold Pfister form $\pi$ is supreme, it is actually sufficient to check that $\pi$ is universal and that anisotropic forms of certain dimensions over $F$ are similar to subforms of $\pi(3.6)$.

We will denote by $\mathcal{S}(n)$ the class of fields of characteristic not 2 which have a supreme $n$-fold Pfister form. Very simple examples of fields with supreme form can be obtained from the following observation: if $F \in \mathcal{S}(n-1)$ then $F((X))$, the field of Laurent series in one variable $X$ over $F$, belongs to $\mathcal{S}(n)$ (3.7). Later we shall obtain examples of an essentially different kind; in fact, any anisotropic Pfister form $\pi$ over the field $F$ becomes a supreme form by scalar extension to a suitable field extension of $F(6.2)$.

If $\pi$ is a supreme $n$-fold Pfister form over $F$, then it is in particular the unique anisotropic $n$-fold Pfister form over $F$ and $\pi$ becomes hyperbolic over every quadratic extension of $F$. Sections 4 and 5 deal with these two weaker conditions on $F$ and $\pi$. 
In Section 4 we investigate when an anisotropic form $\pi$ over $F$ becomes isotropic over any nontrivial 2-extension of $F$. We then say that $F$ is 2-maximal with respect to $\pi$. We obtain equivalent characterizations for 2-maximality (4.2). In particular, when $\pi$ is a Pfister form, we obtain such a characterization in terms of the elements of $F^{\times}$represented by the pure part of $\pi$ (4.4). In (6.3) we shall see that a field can be simultaneously 2-maximal with respect to an arbitrarily large number of $n$-fold Pfister forms, provided that $n \geq 2$.

We will denote by $\mathcal{H}(n)$ the class of fields of characteristic not 2 which have a unique $n$-fold Pfister form. Suppose that $F \in \mathcal{H}(n)$. Then $\bar{I}^{n} F$ can be identified uniquely with $\mathbb{F}_{2}$. Hence the multiplication of forms induces an $\mathbb{F}_{2}$-bilinear pairing $\bar{I}^{i} F \times \bar{I}^{n-i} F \longrightarrow \mathbb{F}_{2}$ for any $0 \leq i \leq n$. If for a certain $i$ this pairing is nondegenerate, then there is a natural duality between $\bar{I}^{i} F$ and $\bar{I}^{n-i} F$. Further, if $F \in \mathcal{H}(2)$ then identification of $\bar{I} F$ with $F^{\times} / F^{\times^{2}}$ turns the pairing $\bar{I} F \times \bar{I} F \longrightarrow \mathbb{F}_{2}$ into the map $h$ defined above.

We will denote by $\mathcal{D}(n)$ the class of nonreal fields $F \in \mathcal{H}(n)$ for which the above pairings are all nondegenerate. In Section 5 we study the class $\mathcal{D}(n)$ and compare it with $\mathcal{S}(n)$. Trivially one has $\mathcal{S}(1)=\mathcal{D}(1)$. In the process of showing that $\mathcal{S}(2)=\mathcal{D}(2)$, we obtain further descriptions of the fields in this class; this is done in Theorem 5.6 which reformulates some of the crucial observations of Fröhlich in [6], combining them with a result of Kozioł in [10]. We prove that this class is closed under quadratic field extensions (5.9). The inclusion $\mathcal{S}(n) \subset \mathcal{D}(n)$ remains valid at least when going up to $n=3$ (5.4). However, we then have a proper inclusion, as we shall see in (6.8).

The core of Section 6 is a fairly general construction of fields having certain properties with respect to quadratic forms (6.1). This construction is applied to produce several examples which illustrate the results of Sections $3-5$. It further allows us to give examples for any $n \geq 3$ of nonreal fields with a single anisotropic $n$-fold Pfister form and with $u$-invariant equal to $\infty$ or to any even number greater than or equal to $2^{n}(6.5)$. This is certainly not surprising in view of Merkurjev's $u$-invariant construction, which actually inspired (6.1); the case $n=3$ has been studied before by Meurer [14].

The last two sections give applications of the results obtained so far to the study of two particular field invariants. In Section 7 we consider the 2-symbol length $\lambda_{2}(F)$ and inquire about the range of its values for fields with a supreme $n$-fold Pfister form. Using the construction given in Section 6 we give new examples of nonreal fields $F$ where $\bar{I}^{n} F \neq 0$ for a certain $n>0$ and where the value of $\lambda_{2}(F)$ is determined (7.4).

In the final section we assume that $F$ is nonreal and study the condition that the level form of $F$ is supreme, and further the weaker condition that $F$ is 2-maximal with respect to the level form. As fields with supreme form 
$2^{n} \times\langle 1\rangle$ exist for any $n \geq 0$ (8.6), we obtain new examples of nonreal fields $F$ of prescribed level $s(F)$ and with particular properties concerning the number of squares necessary to express elements and their negatives as sums of squares in $F(8.2)$. We further give examples showing that a field with supreme form $\langle\langle 1,1\rangle\rangle$ may have finite or infinite square class number (8.10).

\section{Preliminaries}

A comprehensive introduction to the algebraic theory of quadratic forms may be found in the books of Lam [12] and Scharlau [19]. We will generally follow the terminology established there, but we recall some of it.

A diagonalized quadratic form over $F$ with coefficients $a_{1}, \ldots, a_{m} \in F^{\times}$is denoted by $\left\langle a_{1}, \ldots, a_{m}\right\rangle$. An $m$-fold Pfister form is a quadratic form of the shape $\left\langle 1, a_{1}\right\rangle \otimes \cdots \otimes\left\langle 1, a_{m}\right\rangle$, written as $\left\langle\left\langle a_{1}, \ldots, a_{m}\right\rangle\right\rangle$ for short.

Let $\varphi$ be a quadratic form over $F$. By $D_{F}(\varphi)$ one denotes the set of nonzero elements of $F$ represented by $\varphi$. If $D_{F}(\varphi)=F^{\times}$then $\varphi$ is called universal. Further, $\varphi$ is called multiplicative if either $\varphi$ is hyperbolic or $\varphi$ is anisotropic and such that $a \varphi \cong \varphi$ holds for every $a \in D_{F}(\varphi)$; in this case $D_{F}(\varphi)$ is a subgroup of $F^{\times}$. Pfister forms are multiplicative [19, Lemma 4.10.4.]. We will frequently use the following fact:

2.1 Lemma. Suppose that $\varphi$ is universal and multiplicative. Then any quadratic form over $F$ which is similar to a subform of $\varphi$ is itself a subform of $\varphi$.

Proof. Let $\psi$ be a form over $F$, similar to a subform of $\varphi$. Then there exists $a \in F^{\times}$such that $\psi$ is a subform of $a \varphi$. As $\varphi$ is universal and multiplicative, $a \varphi$ is equal to $\varphi$, whence $\psi$ is a subform of $\varphi$.

If $K / F$ is a field extension, then we denote by $\varphi_{K}$ the quadratic form over $K$ obtained from $\varphi$ by scalar extension from $F$ to $K$. Suppose that $\varphi$ is of dimension at least 2 . Then we denote by $F(\varphi)$ the function field of the projective quadric defined by $\varphi$ over $F$. Up to $F$-isomorphism, the field $F(\varphi)$ depends only on the similarity class of $\varphi$. Note that $\varphi$ has a nontrivial zero over $F(\varphi)$, i.e. $\varphi_{F(\varphi)}$ is isotropic. Furthermore, $\varphi_{F(\varphi)}$ is hyperbolic if and only if $\varphi$ is hyperbolic or similar to a Pfister form [19, Theorem 4.5.4.(i)]. In case $\operatorname{dim}(\varphi) \leq 1$ we define $F(\varphi):=F$.

Suppose that $v$ is a discrete valuation on $F$. Let $u \in F^{\times}$be a uniformizer. Then any quadratic form over $F$ can be written as an orthogonal sum $\varphi_{1} \perp$ $u \varphi_{2}$ where the forms $\varphi_{1}$ and $\varphi_{2}$ each have a diagonalization over $F$ with only units as entries. If $\varphi$ is a quadratic form over $F$ having a diagonalization 
$\left\langle a_{1}, \ldots, a_{m}\right\rangle$, where $a_{1}, \ldots, a_{m} \in F^{\times}$are units, then we denote the form $\left\langle\bar{a}_{1}, \ldots, \bar{a}_{m}\right\rangle$ over the residue field $\bar{F}$ by $\bar{\varphi}$.

Recall that $v$ is said to be 2-henselian if $v$ has a unique extension to the quadratic closure of $F$.

2.2 Lemma. Suppose that $v$ is a 2-henselian discrete valuation on $F$ with residue field $\bar{F}$ of characteristic different from 2 . Let $u \in F$ be a uniformizer. Let $\varphi_{1}$ and $\varphi_{2}$ be quadratic forms over $F$ which have a diagonalization with only units as entries. The form $\varphi_{1} \perp u \varphi_{2}$ is anisotropic over $F$ if and only if the residue forms $\bar{\varphi}_{1}$ and $\bar{\varphi}_{2}$ over $\bar{F}$ are anisotropic.

Proof. This is proven in [12, Ch. VI, Proposition 1.9] under the stronger hypothesis that $F$ is complete with respect to $v$. However, the proof just relies on the fact that $u$ induces an isomorphism between $W(F)$ and $W(\bar{F})[\mathbb{Z} / 2 \mathbb{Z}]$, mapping the Witt equivalence class of $\varphi_{1} \perp u \varphi_{2}$ to the couple of Witt equivalence classes of $\bar{\varphi}_{1}$ and of $\bar{\varphi}_{2}$. This hypothesis is satisfied as soon as the valuation $v$ is 2-henselian, as explained in [22, p. 488].

\section{$3 \quad$ Fields with supreme forms}

Let $\varphi$ be a quadratic form over $F$. We say that $\varphi$ is supreme if $\varphi$ is anisotropic and if every anisotropic form over $F$ is similar to a subform of $\varphi$. If $\varphi$ is supreme then it is in particular an anisotropic form of maximal dimension over $F$, so $F$ must be a nonreal field with $u(F)=\operatorname{dim}(\varphi)$.

3.1 Examples. (1) The form $\langle 1\rangle$ over $F$ is supreme if and only if $F$ is quadratically closed.

(2) For $a \in F^{\times}$, the form $\langle\langle a\rangle\rangle=\langle 1, a\rangle$ over $F$ is supreme if and only if $F$ is a nonreal field with exactly two square classes, represented by 1 and $-a$. In particular, any finite field of odd characteristic has a supreme 1-fold Pfister form.

(3) Let $n$ be a positive integer. Over $\mathbb{C}\left(\left(X_{1}\right)\right) \ldots\left(\left(X_{n}\right)\right)$, the iterated power series field in $n$ variables over the field of complex numbers, the Pfister form $\left\langle\left\langle X_{1}, \ldots, X_{n}\right\rangle\right\rangle$ is supreme.

3.2 Proposition. Suppose that the form $\varphi$ is supreme. Then $\varphi$ is a Pfister form and every anisotropic form over $F$ is a subform of $\varphi$.

Proof. Since $\varphi$ is supreme, every quadratic form over $F$ of dimension greater than $\operatorname{dim}(\varphi)$ is isotropic. Hence we may choose an anisotropic Pfister form $\pi$ over $F$ of maximal dimension. This choice implies that for every $b \in F^{\times}$the Pfister form $\pi \otimes\langle\langle-b\rangle\rangle$ is hyperbolic, i.e. $b \pi \cong \pi$. Hence $\pi$ must be universal. 
Since the form $\varphi$ is supreme, it contains a subform which is similar to $\pi$ and this can only be $\pi$ itself. On the other hand, as a universal form $\pi$ cannot be a proper subform of any anisotropic form. Therefore $\varphi$ and $\pi$ are equal. Now we know that $\varphi$ is a supreme, universal Pfister form and conclude with (2.1) that every anisotropic form over $F$ is a subform of $\varphi$.

3.3 Corollary. The form $\varphi$ is supreme over $F$ if and only if $F$ is nonreal with finite u-invariant and $\varphi$ is the unique universal anisotropic form over $F$.

Proof. Suppose that $\varphi$ is supreme. Hence $F$ is nonreal with $u(F)=\operatorname{dim}(\varphi)<$ $\infty$. Further $\varphi$ is universal and anisotropic. Let now $\psi$ be any universal anisotropic form over $F$. By (3.2), $\psi$ is a subform of $\varphi$. Since a universal form cannot be a proper subform of an anisotropic form, $\psi$ must be equal to $\varphi$. This shows that $\varphi$ is the only universal anisotropic form over $F$.

Suppose now that $F$ is nonreal with $u(F)<\infty$. Then every anisotropic form over $F$ is contained in a universal anisotropic form. So, if $\varphi$ is actually the only universal anisotropic form over $F$ then every anisotropic form over $F$ is contained in $\varphi$, which means that $\varphi$ is supreme.

3.4 Remark. In view of the last statement, it is worth pointing out that a nonreal field may have a unique universal anisotropic form without being of finite $u$-invariant. To obtain such an example one may start with $F$ nonreal and such that no anisotropic universal forms over $F$ exists (e.g. $F$ is the direct limit of the fields $\left.\mathbb{C}\left(\left(X_{1}\right)\right) \ldots\left(\left(X_{i}\right)\right), i \in \mathbb{N}\right)$. By [2, Théorème 8.4] there exists a field extension $K / F$ together with an element $r \in K^{\times}$such that the form $\langle 1,-r\rangle$ is anisotropic and universal over $K$ and such that the homomorphism $W(F) \rightarrow W(K)$ defined by scalar extension gives an exact sequence $0 \rightarrow W(F) \rightarrow W(K) \rightarrow \mathbb{Z} / 2 \mathbb{Z} \rightarrow 0$. It is then easy to conclude that $K$ is nonreal with $u(K)=u(F)=\infty$ and that $\langle 1,-r\rangle$ is the unique universal anisotropic form over $K$.

Whenever a quadratic form over $F$ is supreme, it is unique with this property (3.3), so we may refer to it as the supreme form of $F$.

3.5 Proposition. Let $n \geq 1$. Suppose that $F$ has a supreme $n$-fold Pfister form $\pi$. Then

(a) $F$ is nonreal with $u(F)=2^{n}$,

(b) $I^{n+1} F=0$,

(c) $\pi$ is the unique anisotropic $n$-fold Pfister form over $F$,

(d) every anisotropic form in $I^{n-1} F$ is either equal to $\pi$ or similar to an $(n-1)$-fold Pfister form, 
(e) for every nonhyperbolic form $\varphi$ over $F$ there exists a Pfister form $\psi$ over $F$ such that $\varphi \otimes \psi$ is Witt equivalent to $\pi$; moreover, if $\varphi \in I^{i} F$ and $\operatorname{dim}(\varphi)<2^{i+1}$ for some $i \leq n$ then $\psi$ is an $(n-i)$-fold Pfister form.

Proof. (a) We already know that $F$ is nonreal and that $u(F)=\operatorname{dim}(\pi)=2^{n}$.

(b) Since $\pi$ is supreme, every $(n+1)$-fold Pfister form over $F$ is hyperbolic. Hence the ideal $I^{n+1} F$, generated by the $(n+1)$-fold Pfister forms, is zero.

(c) Any anisotropic $n$-fold Pfister form over $F$ is a subform of $\pi$ by (3.2) and of dimension $2^{n}$, hence equal to $\pi$.

(d) Suppose that $\varphi$ is an anisotropic form in $I^{n-1} F$, not equal to $\pi$. Then $\varphi$ is a proper subform of $\pi$, by (3.2), i.e. we may decompose $\pi$ into $\varphi \perp \psi$ where $\psi$ is some anisotropic form over $F$. Now $\psi$ belongs to $I^{n-1} F$, as $\varphi$ and $\pi$ do. By the Arason-Pfister-Hauptsatz [19, Theorem 4.5.6.], $\varphi$ and $\psi$ are each of dimension at least $2^{n-1}$. Since $\operatorname{dim}(\varphi \perp \psi)=2^{n}$, we conclude that $\operatorname{dim}(\varphi)=\operatorname{dim}(\psi)=2^{n-1}$. A well-known corollary of the Arason-PfisterHauptsatz then says that $\varphi$ is similar to an $(n-1)$-fold Pfister form.

(e) Let $\varphi$ be an anisotropic form over $F$. We choose an $m$-fold Pfister form $\psi$ over $F$ such that $\varphi \otimes \psi$ is not hyperbolic and where $m \geq 0$ is as large as possible. Let $\rho$ denote the anisotropic part of $\varphi \otimes \psi$. By the choice of $\psi$ and $m$, the form $\varphi \otimes \psi \otimes\langle\langle a\rangle\rangle$ is hyperbolic for any $a \in F^{\times}$. Thus for every $a \in F^{\times}$one has $\rho \cong a \rho$, in particular $\rho$ is universal. Since $\pi$ is the unique universal anisotropic form over $F$ it follows that $\rho$ is equal to $\pi$, whence $\varphi \otimes \psi$ is Witt equivalent to $\pi$.

Suppose now that we had $\varphi \in I^{i} F$ with $\operatorname{dim}(\varphi)<2^{i+1}$. It follows that $2^{n}=\operatorname{dim}(\pi) \leq \operatorname{dim}(\varphi \otimes \psi)<2^{m+i+1}$ and thus $m \geq n-i$. Since $\varphi \otimes \psi$ is a nonhyperbolic form in $I^{m+i} F$ and since $I^{n+1} F=0$ by $(b)$, we need to have $m=n-i$. Hence $\psi$ is an $(n-i)$-fold Pfister form.

3.6 Proposition. Let $\pi$ be an anisotropic $n$-fold Pfister form over $F$ where $n \geq 1$. The following are equivalent:

(i) $\pi$ is supreme,

(ii) every anisotropic form over $F$ of dimension at least $2^{n-1}+1$ is similar to a subform of $\pi$,

(iii) $\pi$ is universal and every anisotropic form over $F$ of dimension at most $2^{n-1}+1$ is similar to a subform of $\pi$.

Proof. The implication $(i \Rightarrow i i)$ is clear from the definition of a supreme form.

(ii $\Rightarrow$ iii) Suppose that every anisotropic quadratic form $\varphi$ over $F$ with $\operatorname{dim}(\varphi) \geq 2^{n-1}+1$ is similar to a subform of $\pi$. Then $\pi \perp\langle-a\rangle$ is isotropic for any $a \in F^{\times}$, thus $\pi$ is universal. 
Let now $\psi$ be an anisotropic form over $F$ with $\operatorname{dim}(\psi) \leq 2^{n-1}+1$. We want to show that $\psi$ is a subform of $\pi$. If $\operatorname{dim}(\psi)=2^{n-1}+1$ then this is already clear from the hypothesis and from (2.1). Suppose now that $\operatorname{dim}(\psi) \leq 2^{n-1}$. Let $\varphi$ denote the anisotropic part of the form $\pi \perp-\psi$. We have $\operatorname{dim}(\varphi) \geq$ $\operatorname{dim}(\pi)-\operatorname{dim}(\psi) \geq 2^{n-1}$. If these are equalities then the forms $\pi$ and $\psi \perp \varphi$ both have dimension $2^{n}$ and since they are Witt equivalent they are also isometric, so $\psi$ is a subform of $\pi$. In the remaining case we have $\operatorname{dim}(\varphi)>$ $2^{n-1}$. Then, by the hypothesis and by (2.1), there exists a form $\psi^{\prime}$ over $F$ such that $\varphi \perp \psi^{\prime}$ is equal to $\pi$, hence Witt equivalent to $\varphi \perp \psi$. By Witt cancellation, $\psi$ and $\psi^{\prime}$ are Witt equivalent and, since both forms are anisotropic, they are isometric. Thus $\psi$ is a subform of $\pi$.

$($ iii $\Rightarrow i)$ We suppose that $\pi$ is not supreme. Then there exists an anisotropic form $\varphi$ over $F$ which is not a subform of $\pi$. We choose $\varphi$ of the least possible dimension under this condition. We may decompose $\varphi$ into $\varphi^{\prime} \perp\langle a\rangle$ where $a \in F^{\times}$and where $\varphi^{\prime}$ is a form over $F$ with $\operatorname{dim}\left(\varphi^{\prime}\right)=$ $\operatorname{dim}(\varphi)-1$. By the choice of $\varphi$, it is possible to write $\pi \cong \varphi^{\prime} \perp \psi^{\prime}$ with a form $\psi^{\prime}$ over $F$. Since $\varphi$ is not a subform of $\pi$, the form $\psi^{\prime}$ cannot represent $a$, i.e. the form $\psi:=\psi^{\prime} \perp\langle-a\rangle$ is anisotropic. Now, if we had $\psi \perp \varphi^{\prime \prime} \cong \pi$ for some form $\varphi^{\prime \prime}$ over $F$, then $\varphi^{\prime}$ would be isometric to $\langle-a\rangle \perp \varphi^{\prime \prime}$, in particular, $\varphi^{\prime}$ would represent $-a$; this is impossible since $\varphi \cong \varphi^{\prime} \perp\langle a\rangle$ is anisotropic. Therefore $\psi$ is not a subform of $\pi$. From our choice of $\varphi$ we deduce that $\operatorname{dim}(\psi) \geq \operatorname{dim}(\varphi)$. Since $\operatorname{dim}(\varphi)=\operatorname{dim}\left(\varphi^{\prime}\right)+1, \operatorname{dim}(\psi)=\operatorname{dim}\left(\psi^{\prime}\right)+1$ and $\operatorname{dim}\left(\varphi^{\prime}\right)+\operatorname{dim}\left(\psi^{\prime}\right)=\operatorname{dim}(\pi)=2^{n}$, we conclude that $\operatorname{dim}(\varphi) \leq 2^{n-1}+1$. By (2.1), either $\pi$ is not universal or $\varphi$ is not even similar to a subform of $\pi$. So (iii) is not satisfied. This shows the implication $(i i i \Rightarrow i)$.

3.7 Proposition. Suppose that $F$ carries a 2-henselian valuation $v$ with residue field $\bar{F}$ of characteristic different from 2 . Let $u \in F^{\times}$be a uniformizer and $a_{1}, \ldots, a_{n-1} \in F^{\times}$units, $n>1$. The Pfister form $\left\langle\left\langle u, a_{1}, \ldots, a_{n-1}\right\rangle\right\rangle$ over $F$ is supreme if and only if the Pfister form $\left\langle\left\langle\bar{a}_{1}, \ldots, \bar{a}_{n-1}\right\rangle\right\rangle$ over $\bar{F}$ is supreme. In particular, $F \in \mathcal{S}(n)$ if and only of $\bar{F} \in \mathcal{S}(n-1)$.

Proof. The proof is straightforward, using (2.2).

From the proposition and from $(3.1,(2))$ it is clear that any nondyadic local field has a supreme 2-fold Pfister form. In (5.8) we will obtain this for any local field.

3.8 Remark. Let $n \geq 1$. Let $\left(F_{i}\right)_{0 \leq i \leq n}$ be a sequence of fields such that $F_{0}$ is finite and $F_{i}$ is complete with respect to a discrete valuation with residue field $F_{i-1}$ for $1 \leq i \leq n$. Then $F_{n}$ is called an $n$-dimensional local field. From the last proposition and from (5.8) one sees that $F_{n}$ has an $(n+1)$ fold supreme Pfister form provided that $\operatorname{char}\left(F_{1}\right) \neq 2$. The author ignores 
whether this is equally true in the case where there is a number $m<n$ such that $\operatorname{char}\left(F_{i}\right)=2$ for $0 \leq i \leq m$ and $\operatorname{char}\left(F_{i}\right)=0$ for $m<i \leq n$.

\section{$4 \quad$ 2-Maximality}

A field extension of $F$ which can be embedded into a quadratic closure of $F$ is called a 2-extension. Let $\varphi$ be a quadratic form over $F$. We say that $F$ is 2 -maximal with respect to $\varphi$ if $\varphi$ is anisotropic and if $\varphi_{K}$ is isotropic for every nontrivial 2-extension $K / F$.

4.1 Proposition. For any anisotropic form $\varphi$ over $F$ there is a 2-extension $K / F$ such that $K$ is 2-maximal with respect to $\varphi_{K}$.

Proof. Let $F_{q}$ denote a quadratic closure of $F$. Among the subfields of $F_{q}$ which contain $F$ we choose $K$ maximal under the condition that $\varphi_{K}$ is anisotropic. Since $F_{q}$ is also a quadratic closure of $K$, the choice of $K$ implies that $\varphi_{L}$ is isotropic for any nontrivial 2-extension $L / K$. Hence $K$ is 2-maximal with respect to $\varphi_{K}$.

4.2 Theorem. Let $\varphi$ be an anisotropic form over $F$. The following conditions are equivalent:

(i) $F$ is 2-maximal with respect to $\varphi$,

(ii) for every extension $K / F$ such that $\varphi_{K}$ is anisotropic, $F$ is quadratically closed in $K$,

(iii) every anisotropic binary form over $F$ is similar to a subform of $\varphi$.

Proof. $(i \Rightarrow i i)$ Let $K / F$ be an extension such that $\varphi_{K}$ is anisotropic. Let $F^{\prime}$ denote the quadratic closure of $F$ in $K$. Then $F^{\prime} / F$ is a 2-extension and $\varphi_{F^{\prime}}$ is anisotropic. Thus, if $F$ is 2 -maximal with respect to $\varphi$ then $F^{\prime}=F$, i.e. $F$ is quadratically closed in $K$.

$(i i \Rightarrow$ iii $)$ Let $\beta$ be an anisotropic binary form over $F$. Then $\beta$ is similar to $\langle 1,-a\rangle$ for some $a \in F^{\times} \backslash F^{\times 2}$. Condition (ii) implies that $\varphi_{F(\sqrt{a})}$ is isotropic. By [19, Lemma 3.5.1.], $\varphi$ then contains a subform similar to $\langle 1,-a\rangle$, hence similar to $\beta$.

$($ iii $\Rightarrow i)$ Let $K / F$ be a nontrivial 2-extension. By basic Galois theory, $K$ then contains a quadratic extension of $F$. Hence there is some $a \in F^{\times}$ which is a square in $K$ but not in $F$. In other words, the binary form $\langle 1,-a\rangle$ is anisotropic over $F$ but isotropic over $K$. By the hypothesis (iii), over $F$ the form $\langle 1,-a\rangle$ is similar to a subform of $\varphi$. Therefore $\varphi_{K}$ is isotropic. 
4.3 Corollary. Let $\varphi$ be a quadratic form over $F$. If $\varphi$ is supreme then $F$ is 2-maximal with respect to $\varphi$.

Proof. If $\varphi$ is supreme then condition (iii) in (4.2) is satisfied.

4.4 Corollary. Let $\pi$ be an anisotropic Pfister form over $F$ and let $\pi^{\prime}$ denote the pure subform of $\pi$. The field $F$ is 2-maximal with respect to $\pi$ if and only if $D_{F}\left(\pi^{\prime}\right)=F^{\times} \backslash-F^{\times 2}$.

Proof. By the definition of the pure subform, $\pi$ is equal to $\langle 1\rangle \perp \pi^{\prime}$. Since $\pi$ is anisotropic, $\pi^{\prime}$ cannot represent -1 , thus we have $D_{F}\left(\pi^{\prime}\right) \subset F^{\times} \backslash-F^{\times 2}$. Equality $D_{F}\left(\pi^{\prime}\right)=F^{\times} \backslash-F^{\times 2}$ means that $\langle 1, a\rangle$ is a subform of $\pi$ for every $a \in F^{\times} \backslash-F^{\times 2}$. Since $\pi$ is multiplicative the last is equivalent to condition (iii) in (4.2) for $\varphi=\pi$. Now the statement follows using the equivalence $(i \Leftrightarrow i i i)$ of $(4.2)$.

The field $F$ is called real euclidean if $F^{\times^{2}}$ is an ordering of $F$, i.e. if $F$ is real and $F^{\times}=F^{\times^{2}} \cup-F^{\times^{2}}$.

4.5 Corollary. Let $F$ be real and $\pi$ a nontorsion $n$-fold Pfister form over $F$, where $n \geq 1$. The field $F$ is 2-maximal with respect to $\pi$ if and only if $F$ is real euclidean and $\pi$ is equal to $2^{n} \times\langle 1\rangle$.

Proof. Suppose that $F$ is real euclidean. Then every anisotropic binary form over $F$ is similar to $\langle 1,1\rangle$, a subform of $2^{n} \times\langle 1\rangle$. Thus, by (4.2) $F$ is 2 maximal with respect to $2^{n} \times\langle 1\rangle$, which is an anisotropic nontorsion $n$-fold Pfister form over $F$.

For the converse, suppose that $F$ is 2-maximal with respect to $\pi$. As $\pi$ is a nontorsion Pfister form, Pfister's local-global principle [19, Theorem 3.6.2.] shows that there exists a real closure $K / F$ for which $\pi_{K}$ is anisotropic. Then, by the theorem, $F$ is quadratically closed in $K$, showing that $F$ is real euclidean. In particular, $2^{n} \times\langle 1\rangle$ is the unique anisotropic $n$-fold Pfister form over $F$, hence equal to $\pi$.

We will show in (6.3) that $F$ can be 2-maximal with respect to many different $n$-fold Pfister forms simultaneously when $n \geq 2$.

\section{Comparison of the classes $\mathcal{S}(n)$ and $\mathcal{D}(n)$}

Let $n$ be a positive integer. We denote by $\mathcal{H}(n)$ the class of fields of characteristic different from 2 having a unique anisotropic $n$-fold Pfister form. Note that the class $\mathcal{H}(n)$ is not closed under quadratic field extensions. For every $n \geq 0$ one has $\mathbb{R} \in \mathcal{H}(n)$ but $\mathbb{C} \notin \mathcal{H}(n)$, for example. A less trivial 
phenomenon is the existence, for any $n \geq 2$, of field extensions $K / F$ where $F$ is nonreal and belongs to $\mathcal{H}(n)$ while $K$ has three different anisotropic $n$ fold Pfister forms. The different possibilities for the number of $n$-fold Pfister forms over $K$ where $K / F$ is a quadratic field extension with $F \in \mathcal{H}(n)$ have been determined by Kozioł in [10, Theorem 1]. From his result we easily derive the following consequence:

5.1 Proposition. Suppose that $F \in \mathcal{H}(n)$. Let $\pi$ denote the unique anisotropic $n$-fold Pfister form over $F$. All quadratic extensions of $F$ belong to $\mathcal{H}(n)$ if and only if $F$ is nonreal and 2-maximal with respect to $\pi$.

Proof. By [10, Theorem 1], if $K / F$ is a quadratic extension then $K$ has a unique $n$-fold Pfister form if and only if $F$ is nonreal and $\pi_{K}$ is hyperbolic. By (4.2), $\pi_{K}$ is hyperbolic for every quadratic extension $K / F$ if and only if $F$ is 2-maximal with respect to $\pi$.

Suppose that $F \in \mathcal{H}(n)$. As the group $\bar{I}^{n} F$ consists of two elements it can be uniquely identified with $\mathbb{F}_{2}$. Thus for every $0 \leq i \leq n$ multiplication of forms in $I^{i} F$ with forms in $I^{n-i} F$ induces a canonical $\mathbb{F}_{2}$-bilinear pairing $\bar{I}^{i} F \times \bar{I}^{n-i} F \longrightarrow \mathbb{F}_{2}$. Trivially, this pairing is nondegenerate for $i=0$ and $i=n$. Further, for $F \in \mathcal{H}(2)$ the canonical pairing $\bar{I} F \times \bar{I} F \longrightarrow \mathbb{F}_{2}$ is the same as the map $h$ defined at the beginning of the introduction if one identifies $\bar{I} F$ with $F^{\times} / F^{\times^{2}}$ via the discriminant (signed determinant).

5.2 Proposition. Let $F$ be a nonreal field with a unique anisotropic $n$-fold Pfister form $\pi$. The following are equivalent:

(i) for every $1 \leq i<n$ the canonical $\mathbb{F}_{2}$-bilinear pairing $\bar{I}^{i} F \times \bar{I}^{n-i} F \longrightarrow \mathbb{F}_{2}$ is nondegenerate,

(ii) for every $1 \leq i<n$ and every form $\varphi \in I^{i} F \backslash I^{i+1} F$ there exists an element $a \in F^{\times}$such that $\varphi \otimes\langle\langle a\rangle\rangle \notin I^{i+2} F$,

(iii) for every $1 \leq i<n$ and every form $\varphi \in I^{i} F \backslash I^{i+1} F$ there exists an $(n-i)$-fold Pfister form $\psi$ such that $\varphi \otimes \psi$ is Witt equivalent to $\pi$.

Proof. $(i \Rightarrow i i)$ Let $\varphi$ be a form in $I^{i} F \backslash I^{i+1} F$, where $1 \leq i<n$. Condition (i) implies that $\varphi \cdot I^{n-i} F \not \subset I^{n+1} F$ and therefore $\varphi \cdot I F \not \subset \bar{I}^{i+2} F$. Hence there exists $a \in F^{\times}$such that $\varphi \otimes\langle\langle a\rangle\rangle \notin I^{i+2} F$.

(ii $\Rightarrow$ iii) Let $\varphi$ be a form in $I^{i} F \backslash I^{i+1} F$, where $1 \leq i<n$. By repeated application of $(i i)$, there are elements $a_{i+1}, \ldots, a_{n} \in F^{\times}$such that the form $\varphi \otimes\left\langle\left\langle a_{i+1}, \ldots, a_{n}\right\rangle\right\rangle$ belongs to $I^{n} F \backslash I^{n+1} F$; then this form is Witt equivalent to $\pi$, the only anisotropic form in $I^{n} F$.

$($ iii $\Rightarrow i)$ This is clear. 
We denote by $\mathcal{D}(n)$ the class of nonreal fields in $\mathcal{H}(n)$ for which the equivalent conditions in (5.2) hold. We want to compare this class with $\mathcal{S}(n)$, the class of fields with a supreme $n$-fold Pfister form.

For $n=1$ the conditions in (5.2) are empty, so $\mathcal{D}(1)$ contains just the nonreal fields in $\mathcal{H}(1)$. Hence $\mathcal{D}(1)$ is the class of all nonreal fields of characteristic different from 2 with exactly two square classes and therefore coincides with $\mathcal{S}(1)$.

5.3 Proposition. Suppose that $F \in \mathcal{S}(n)$. Then $F$ is a nonreal field in $\mathcal{H}(n)$ and the canonical pairing $\bar{I} F \times \bar{I}^{n-1} F \longrightarrow \mathbb{F}_{2}$ is nondegenerate.

Proof. We already know that $F$ must be nonreal $(3.5$, a) and that $F \in \mathcal{H}(n)$ $(3.5, \mathrm{c})$. By $(3.5, \mathrm{~d})$, for $i=n-1$ any nontrivial class in $\bar{I}^{i} F$ is represented by an $i$-fold Pfister form, thus by a form in $I^{i} F$ of dimension smaller than $2^{i+1}$. The same holds trivially for $i=1$ as well. Using $(3.5, \mathrm{e})$, we conclude that the pairing $\bar{I} F \times \bar{I}^{n-1} F \longrightarrow \mathbb{F}_{2}$ is nondegenerate.

5.4 Corollary. For $1 \leq n \leq 3$ the class $\mathcal{S}(n)$ is contained in $\mathcal{D}(n)$.

5.5 Question. Does the inclusion $\mathcal{S}(n) \subset \mathcal{D}(n)$ also hold for $n>3$ ?

The following theorem shows that $\mathcal{S}(2)=\mathcal{D}(2)$ and further contains some of the crucial observations made by Fröhlich in [6]. We want to provide a complete proof, even if most ideas in it originate from the articles of Fröhlich [6] and Kaplansky [9].

5.6 Theorem (Fröhlich). The following conditions on $F$ are equivalent:

(i) $F \in \mathcal{S}(2)$,

(ii) $F \in \mathcal{D}(2)$,

(iii) $F$ and all quadratic extensions of $F$ belong to $\mathcal{H}(2)$,

(iv) $F$ is nonreal, has a unique anisotropic 2-fold Pfister form and is 2maximal with respect to this form,

(v) $F$ is nonreal and not quadratically closed, and for any $a \in F^{\times} \backslash F^{\times^{2}}$ the group $D_{F}(\langle 1,-a\rangle)$ has index 2 in $F^{\times}$.

Proof. $(i \Rightarrow i i)$ By (5.4) we have $\mathcal{S}(2) \subset \mathcal{D}(2)$.

$(i i \Rightarrow v)$ Suppose that $F \in \mathcal{D}(2)$. Then $F$ is nonreal and not quadratically closed and the pairing $h: F^{\times} / F^{\times^{2}} \times F^{\times} / F^{\times^{2}} \rightarrow \mathbb{F}_{2}$ defined in the introduction is $\mathbb{F}_{2}$-bilinear and nondegenerate. Therefore, for any $a \in F^{\times} \backslash F^{\times^{2}}$ 
the map $F^{\times} \rightarrow \mathbb{F}_{2}, b \mapsto h\left(a F^{\times^{2}}, b F^{\times^{2}}\right)$ is a surjective homomorphism, so its kernel, which is $D_{F}(\langle 1,-a\rangle)$, has index 2 in $F^{\times}$.

$(v \Rightarrow i v)$ Assume that $F$ is not quadratically closed and that $D_{F}(\langle 1,-a\rangle)$ has index 2 in $F^{\times}$for any $a \in F^{\times} \backslash F^{\times^{2}}$. Then every anisotropic 1-fold Pfister form over $F$ is contained in exactly one anisotropic 2-fold Pfister form.

Since $F$ is not quadratically closed we may choose $a \in F^{\times} \backslash F^{\times^{2}}$. Let $\pi$ be the anisotropic 2-fold Pfister form over $F$ which contains $\langle 1,-a\rangle$.

Let $b$ be another element of $F^{\times} \backslash F^{\times 2}$. Since $D_{F}(\langle 1,-a\rangle)$ and $D_{F}(\langle 1,-b\rangle)$ are both proper subgroups of $F^{\times}$their union cannot be $F^{\times}$. Hence we may choose $c \in F^{\times}$such that the 2-fold Pfister forms $\langle\langle-a,-c\rangle\rangle$ and $\langle\langle-b,-c\rangle\rangle$ are both anisotropic. However, these 2-fold Pfister forms both contain $\langle 1,-c\rangle$, hence they must be equal. But $\pi$ is the only anisotropic 2-fold Pfister form containing $\langle 1,-a\rangle$. This shows that $\langle 1,-b\rangle$ is contained in $\pi$ and in no other anisotropic 2-fold Pfister form.

This shows that $\pi$ is the only anisotropic 2-fold Pfister form over $F$ and that its pure subform represents all elements of $F^{\times} \backslash-F^{\times^{2}}$. By (4.4) this means that $F$ is 2-maximal with respect to $\pi$.

(iv $\Leftrightarrow i i i)$ This equivalence has been established in (5.1).

$(i v \Rightarrow i)$ Assume that $F$ is nonreal and has a unique anisotropic 2-fold Pfister form $\pi$ and that $F$ is 2-maximal with respect to $\pi$. Let $\pi^{\prime}$ denote the pure subform of $\pi$. Since $F$ is nonreal and since $\pi^{\prime}$ is a proper subform of the anisotropic form $\pi$, we know that $D_{F}\left(\pi^{\prime}\right) \subsetneq D_{F}(\pi)$. (This argument is known as Kneser's Lemma.) On the other hand, we have $D_{F}\left(\pi^{\prime}\right)=F^{\times} \backslash-F^{\times^{2}}$, by (4.4). We conclude that $D_{F}(\pi)=F^{\times}$, i.e. $\pi$ is universal.

Further, if $\varphi$ is an anisotropic form over $F$ with $\operatorname{dim}(\varphi) \leq 3$ then $\varphi$ is similar to a subform of $\pi$. Indeed, for $\operatorname{dim}(\varphi)=1$ this is trivial, if $\operatorname{dim}(\varphi)=2$ then this follows by (4.2) from the fact that $F$ is 2-maximal with respect to $\pi$, and finally, if $\operatorname{dim}(\varphi)=3$ then $\varphi$ is similar to a subform of some anisotropic 2-fold Pfister form over $F$ which by hypothesis must be $\pi$.

Using (3.6) we conclude that $\pi$ is supreme.

5.7 Remark. The conditions $(i v)$ and $(v)$ remain equivalent if the assumption that $F$ be nonreal is removed in each of them. The real fields which then satisfy these (modified) conditions are precisely those which are real euclidean.

5.8 Corollary (Fröhlich). Let $F$ be a local field with $\operatorname{char}(F) \neq 2$. Then $F$ satisfies the equivalent conditions of (5.6). The supreme 2-fold Pfister form of $F$ is the norm form of the unique quaternion division algebra over $F$.

Proof. Every finite extension of $F$ is again a local field and thus belongs to $\mathcal{H}(2)$. Therefore condition (iii) in the theorem holds for $F$. 
We give an alternative argument which covers the entire statement. It is well known that there exists a unique quaternion division algebra $Q$ over $F$ [12, Ch. VI, Theorem 2.10] and that $Q \otimes_{F} K$ is split for every quadratic extension $K / F$ [12, Ch. VI, Lemma 2.14]. Let $\pi$ be the norm form of $Q$. Using the standard correspondence between quaternion algebras and 2-fold Pfister forms (cf. [12, Ch. III, Proposition 2.5]), we conclude that $\pi$ is the unique anisotropic 2-fold Pfister form over $F$ and that $\pi$ becomes hyperbolic over every quadratic extension of $F$. Hence $F$ satisfies condition $(i v)$ and the proof of $(i v \Rightarrow i)$ shows that $\pi$ is the supreme form of $F$.

The various descriptions in (5.6) of the fields in the class $\mathcal{S}(2)=\mathcal{D}(2)$ now make it possible to show, using the Norm Principle, that this class is closed under quadratic extensions.

5.9 Theorem. If $F \in \mathcal{S}(2)$, then $K \in \mathcal{S}(2)$ for any quadratic extension $K / F$.

Proof. Let $K / F$ be a quadratic extension where $F \in \mathcal{S}(2)$. Then all the conditions in (5.6) hold for $F$. In particular condition (iii) tells us that $K \in \mathcal{H}(2)$, i.e. $K$ has a unique anisotropic 2 -fold Pfister form. We will show that $K$ satisfies condition $(v)$ in (5.6), which by (5.6) implies that $K \in \mathcal{S}(2)$. It is clear that $K$ is nonreal and not quadratically closed.

Let $x \in K^{\times}$. We want to show that $D_{K}(\langle 1,-x\rangle)$ has index 2 in $K^{\times}$unless $x$ is a square in $K$. Since $\langle 1,-x\rangle$ is a subform of at most one anisotropic 2 -fold Pfister form over $K$ we know that $D_{K}(\langle 1,-x\rangle)$ has index 1 or 2 in $K^{\times}$.

Now we assume that $D_{K}(\langle 1,-x\rangle)=K^{\times}$and have to show that then $x$ must be a square in $K$. The assumption can be reformulated by saying that $x$ is represented over $K$ by every binary form $\langle 1, y\rangle$ with $y \in K^{\times}$. Having this in particular for all $y \in F^{\times}$we conclude by [5, Norm Principle 2.13.] that $N_{K / F}(x) \in F^{\times}$, the norm of $x$ over $F$, is represented over $F$ by every binary form $\langle 1, a\rangle$ with $a \in F^{\times}$. This means that the form $\left\langle 1,-N_{K / F}(x)\right\rangle$ over $F$ is universal. Since condition $(v)$ in $(5.6)$ holds for $F$, it follows that $N_{K / F}(x) \in F^{\times 2}$. Therefore we have $x \in F^{\times} K^{\times 2}$ [12, Ch. VII, Theorem 3.4].

Hence we may assume that $x \in F^{\times}$. Again by [5, Norm Principle 2.13.], since $\langle 1,-x\rangle$ is universal over $K$, the same form over $F$ represents all nonzero norms of $K / F$. In other terms, if we write $K=F(\sqrt{d})$ with $d \in F^{\times} \backslash F^{\times^{2}}$, then we have $D_{F}(\langle 1,-d\rangle) \subset D_{F}(\langle 1,-x\rangle)$. But condition $(v)$ in (5.6) says that $D_{F}(\langle 1,-d\rangle)$ has index 2 in $F^{\times}$. Hence $D_{F}(\langle 1,-x\rangle)$ is either equal to $F^{\times}$or to $D_{F}(\langle 1,-d\rangle)$. In the first case, by condition $(v)$ in (5.6), $x$ must be a square in $F$, hence also in $K$. Assume now that we are in the case where $D_{F}(\langle 1,-x\rangle)=D_{F}(\langle 1,-d\rangle)$. For any $b \in F^{\times}$, the form $\langle\langle-x, b\rangle\rangle$ over $F$ is hyperbolic if and only if $\langle\langle-d, b\rangle\rangle$ is hyperbolic; at any rate both forms are equal because $F$ has only one anisotropic 2 -fold Pfister form, and we conclude 
that $\langle\langle-x d, b\rangle\rangle$ must be hyperbolic. This shows that $\langle 1,-x d\rangle$ is universal over $F$. By condition $(v)$ in (5.6) this is only possible if $x d \in F^{\times^{2}}$. Hence also in the second case $x$ is a square in $K=F(\sqrt{d})$.

5.10 Question. Let $n \geq 3$. Are the classes $\mathcal{S}(n)$ and $\mathcal{D}(n)$ closed under quadratic extensions?

5.11 Proposition. Let $n>1$ and suppose that $F$ carries a 2-henselian valuation $v$ with residue field $\bar{F}$ of characteristic different from 2 . Then $F \in \mathcal{D}(n)$ if and only if $\bar{F} \in \mathcal{D}(n-1)$.

Proof. Let $u \in F^{\times}$be a uniformizer for $v$. From (2.2) one easily deduces, first, that $F \in \mathcal{H}(n)$ if and only if $\bar{F} \in \mathcal{H}(n-1)$, and second, that $F$ is nonreal if and only if $\bar{F}$ is nonreal.

Assume that $\bar{F} \in \mathcal{D}(n-1)$. To show that $F \in \mathcal{D}(n)$ it is sufficient to check condition $(i i)$ in (5.2). Let $\varphi \in I^{i} F \backslash I^{i+1} F$, where $1 \leq i<n$. Now, $\varphi$ is Witt equivalent to $\varphi_{0} \perp\langle\langle u\rangle\rangle \otimes \varphi_{1}$ where $\varphi_{0}$ and $\varphi_{1}$ are forms over $F$ with diagonalizations with only units as entries. Then we must have $\varphi_{0} \in I^{i} F$ and $\varphi_{1} \in I^{i-1} F$. If now $\varphi_{0} \notin I^{i+1} F$, then $\varphi \otimes\langle\langle-u\rangle$, which is Witt equivalent to $\varphi_{0} \otimes\left\langle\langle-u\rangle\right.$, cannot lie in $I^{i+2} F$. To do the other case, we assume now that $\varphi_{0} \in I^{i+1} F$. Then we have necessarily $\varphi_{1} \notin I^{i} F$, so the residue form $\bar{\varphi}_{1}$ over $\bar{F}$ lies in $I^{i-1} \bar{F} \backslash I^{i} \bar{F}$. Since $\bar{F} \in \mathcal{D}(n-1)$ we conclude with (5.2) that there exists some unit $a \in F^{\times}$such that $\bar{\varphi}_{1} \otimes\langle\langle\bar{a}\rangle\rangle \notin I^{i+1} \bar{F}$. Then over $F$ we have $\langle\langle u\rangle\rangle \otimes \varphi_{1} \otimes\langle\langle a\rangle\rangle \notin I^{i+2} F$, while $\varphi_{0} \otimes\langle\langle a\rangle\rangle \in I^{i+2} F$, and therefore $\varphi \otimes\langle\langle a\rangle\rangle \notin I^{i+2} F$, what we wanted to show.

Assume now that $F \in \mathcal{D}(n)$. Any form over $\bar{F}$ is of the shape $\bar{\varphi}$ where $\varphi$ is a form over $F$ with a diagonalization with only units as entries. Suppose that $\bar{\varphi} \in I^{i} \bar{F} \backslash I^{i+1} \bar{F}$ where $1 \leq i<n-1$. Then we have $\varphi \otimes\langle\langle u\rangle\rangle \in I^{i+1} F \backslash I^{i+2} F$. By our assumption and by (5.2) there is $a \in F^{\times}$such that $\varphi \otimes\langle\langle u, a\rangle\rangle \notin I^{i+3} F$. Further, since $v(u)=1$ we may choose $a$ as a unit. Then it follows that $\bar{\varphi} \otimes\langle\langle\bar{a}\rangle\rangle \notin I^{i+2} \bar{F}$. By $(5.2)$ this shows that $\bar{F} \in \mathcal{D}(n-1)$.

We finish this section with a statement that will be used later to show that $\mathcal{S}(3) \subsetneq \mathcal{D}(3)$. It gives a condition on $F$ which implies that $F \in \mathcal{D}(3) \backslash \mathcal{S}(3)$. That fields subject to this condition exist will be shown in (6.7).

Recall that an Albert form is by definition a 6-dimensional quadratic form of determinant -1 . 
5.12 Proposition. Suppose that there exist an anisotropic 3-fold Pfister form $\pi$ and an anisotropic Albert form $\alpha$ over $F$ such that every anisotropic form over $F$ is similar to a subform of $\pi$ or of $\alpha$. Then

(a) $F$ is nonreal with $u(F)=8$,

(b) there exist exactly three universal anisotropic forms over $F$ and these are $\pi, \alpha$ and some form similar to $\alpha$,

(c) any anisotropic form over $F$ of dimension at most 4 is a subform of $\pi$,

(d) F has no supreme form,

(e) $F \in \mathcal{D}(3) \backslash \mathcal{S}(3)$.

Proof. (a) By hypothesis, every form over $F$ of dimension greater than 8 is isotropic. Thus $F$ is nonreal and $u(F)=\operatorname{dim}(\pi)=8$.

(b) As $\alpha$ has determinant -1 and dimension 6 , it cannot be similar to a subform of any anisotropic 3-fold Pfister form. It follows from the hypothesis that an anisotropic form over $F$ is universal if and only it is similar either to $\pi$ or to $\alpha$.

Since $\pi$ is universal we have $a \pi \cong \pi$ for every $a \in F^{\times}$. In particular $\pi$ is the only anisotropic form in $I^{3} F$. Further for any $a \in F^{\times}$the form $\langle 1, a\rangle \otimes \alpha$ lies in $I^{3} F$, hence it is either hyperbolic or Witt equivalent to $\pi$. Therefore there is at most one form which is similar but not isometric to $\alpha$.

Let $\beta$ denote the anisotropic part of the form $\pi \perp \alpha$. This form lies in $I^{2} F$ and its Clifford algebra is Brauer equivalent to the Clifford algebra of $\alpha$, in particular it is of index 4 . Therefore $\beta$ is of dimension at least 6 and not similar to a subform of $\pi$. From the hypothesis we conclude that $\beta$ is similar to $\alpha$. However, $\beta \perp-\alpha$ is Witt equivalent to $\pi$, so $\alpha$ and $\beta$ are not isometric.

All this together shows that $\pi, \alpha$ and $\beta$ are the only anisotropic universal forms over $F$ and that they are distinct.

(c) Let $\varphi$ be an anisotropic form over $F$. Let $\psi$ denote the anisotropic part of $\pi \perp-\varphi$. Then $\pi$ is Witt equivalent to $\varphi \perp \psi$. Hence $\varphi$ is a subform of $\pi$ if and only if $\psi$ is a subform of $\pi$, if and only if $\operatorname{dim}(\varphi)+\operatorname{dim}(\psi)=\operatorname{dim}(\pi)$. Suppose now that $\varphi$ is of dimension at most 4 but not a subform of $\pi$. Then $\psi$ is not a subform of $\pi$ either and we have $\operatorname{dim}(\psi) \geq \operatorname{dim}(\pi)-\operatorname{dim}(\varphi)+2 \geq 6$. Note that $\pi$ is universal and multiplicative. Thus by (3.2), $\psi$ is not similar to a subform of $\pi$. Hence the hypothesis implies that $\psi$ is similar to $\alpha$. It follows that $\varphi$ is similar to the anisotropic part of $\pi \perp \alpha$, which is $\beta$. This is impossible since $\operatorname{dim}(\beta)=6>\operatorname{dim}(\varphi)$. 
$(d)$ By $(b)$ there is more than one universal anisotropic form over $F$. Therefore $F$ has no supreme form.

(e) We know that $F$ is nonreal and that $\pi$ is the unique anisotropic 3-fold Pfister form over $F$.

Let $\varphi$ be an anisotropic form in $I F \backslash I^{2} F$. Then the signed determinant $d:=d_{ \pm}(\varphi)$ is not a square in $F$. Hence $\langle 1,-d\rangle$ is anisotropic, but not universal by $(b)$. Let $a \in F^{\times}$be such that $\langle\langle-d, a\rangle\rangle$ is anisotropic. Then $\varphi \otimes\langle\langle b\rangle\rangle$ lies in the same nontrivial class of $\bar{I}^{2} F$ as $\langle\langle-d, a\rangle\rangle$, thus $\varphi \otimes\langle\langle a\rangle\rangle \notin$ $I^{3} F$. Let now $\varphi$ be an anisotropic form in $I^{2} F \backslash I^{3} F$. Then $\varphi$ is similar to $\alpha$ or to some 2-fold Pfister form. It follows from $(b)$ and $(c)$ that there exists $a \in F^{\times}$such that $\varphi \otimes\langle 1, a\rangle$ is Witt equivalent to $\pi$ and does therefore not lie in $I^{4} F$. By (5.2) this shows that $F \in \mathcal{D}(3)$. But $F \notin \mathcal{S}(3)$ by $(d)$.

\section{Construction of examples}

Let $\mathcal{C}$ be a class of field extensions of $F$, i.e. a class of fields having $F$ as a subfield. We say that $\mathcal{C}$ is admissible if the following conditions are satisfied:

(i) $\mathcal{C}$ is closed under direct limits,

(ii) if $K \in \mathcal{C}$ and if $K^{\prime}$ is a subfield of $K$ which contains $F$ then $K^{\prime} \in \mathcal{C}$,

(iii) $\mathcal{C}$ is not empty.

Note that, in view of $(i i)$, condition $(i i i)$ means that $F \in \mathcal{C}$.

6.1 Theorem. Let $\mathcal{C}$ be an admissible class of field extensions of $F$. There exists a field $K$ containing $F$ such that $K \in \mathcal{C}$ but $K(\varphi) \notin \mathcal{C}$ for any anisotropic quadratic form $\varphi$ over $K$ of dimension at least 2. Moreover, if $F$ is infinite then $K$ may be chosen of the same cardinality as $F$.

Note that if for example $\mathcal{C}$ is the class of all field extensions of $F$, then we may take for $K$ any quadratically closed field containing $F$; since every quadratic form of dimension at least 2 over $K$ is isotropic, the second condition will be trivially satisfied.

Proof. As a first step we show that every field $F^{\prime} \in \mathcal{C}$ has an extension $F^{\prime \prime} \in \mathcal{C}$ such that for any anisotropic quadratic form $\varphi$ over $F^{\prime}$ of dimension at least 2 , either $\varphi_{F^{\prime \prime}}$ is isotropic or $F^{\prime \prime}(\varphi) \notin \mathcal{C}$. Let $F^{\prime} \in \mathcal{C}$. Let $\mathcal{M}$ be a subset of $W\left(F^{\prime}\right)$. Each nonzero class in $W\left(F^{\prime}\right)$ is represented by some anisotropic form over $F$, uniquely determined up to isometry. In the case where $\mathcal{M}$ is finite, let $\varphi_{1}, \ldots, \varphi_{m}$ be anisotropic forms over $F$ representing the distinct nonzero 
classes in $\mathcal{M}$ and denote by $F^{\prime}(\mathcal{M})$ the function field $F^{\prime}\left(\varphi_{1}\right) \ldots\left(\varphi_{m}\right)$, up to $F$-isomorphism only depending on $\mathcal{M}$. More generally, let $F^{\prime}(\mathcal{M})$ denote the direct limit of all fields $F^{\prime}\left(\mathcal{M}^{\prime}\right)$ where $\mathcal{M}^{\prime} \subset \mathcal{M}$ is finite. By hypothesis $\mathcal{C}$ is closed under direct limits. Thus by Zorn's Lemma, there is a subset $\mathcal{M}$ of $W\left(F^{\prime}\right)$ which is maximal under the condition that $F^{\prime}(\mathcal{M}) \in \mathcal{C}$. We denote the field $F^{\prime}(\mathcal{M})$ by $F^{\prime \prime}$. Let $\varphi$ be an anisotropic form of dimension at least 2 over $F^{\prime}$. If $\varphi_{F^{\prime \prime}}$ is anisotropic, then the Witt equivalence class $[\varphi]$ does not belong to $\mathcal{M}$ and $F^{\prime \prime}(\varphi)$ is $F$-isomorphic to $F^{\prime}(\mathcal{M} \cup[\varphi])$. So, by the maximality of $\mathcal{M}$, we have $F^{\prime \prime}(\varphi) \notin \mathcal{C}$. Moreover, if $F^{\prime}$ is infinite, then the field $F^{\prime \prime}$ constructed here is of the same cardinality as $F^{\prime}$. (Note that the cardinality of $W\left(F^{\prime}\right)$ is at most equal to the cardinality of $F^{\prime}$.)

By the first step, we may now choose a sequence of fields $\left(F_{i}\right)_{i \in \mathbb{N}}$ in $\mathcal{C}$ such that for $i \in \mathbb{N}$ one has $F_{i} \subset F_{i+1}$ and for every anisotropic form $\varphi$ over $F_{i}$ of dimension at least 2 either $\varphi_{F_{i+1}}$ is isotropic or $F_{i+1}(\varphi) \notin \mathcal{C}$. Let $K$ be the union of all the fields $F_{i}$. Since $\mathcal{C}$ is closed under direct limits, $K \in \mathcal{C}$. Let $\varphi$ be an anisotropic form of dimension at least 2 over $K$. Then $\varphi$ is defined over $F_{i}$ for some $i \in \mathbb{N}$. Since $\varphi$ is anisotropic over $F_{i+1}$ we must have $F_{i+1}(\varphi) \notin \mathcal{C}$. As the field $K(\varphi)$ contains $F_{i+1}(\varphi)$, condition $(i i)$ for admissibility shows that $K(\varphi) \notin \mathcal{C}$. Moreover, if $F$ is infinite, the fields $F_{i}$ can be chosen of the same cardinality as $F$; then $K$ also has the same cardinality as $F$.

6.2 Theorem. Let $n \geq 1$ and let $\pi$ be an anisotropic $n$-fold Pfister form over $F$. There exists a field extension $K$ of $F$ such that

(i) the form $\pi_{K}$ is supreme over $K$,

(ii) for every anisotropic form $\psi$ over $F$ of dimension less than or equal to $2^{n-1}$, the form $\psi_{K}$ is anisotropic over $K$,

(iii) for every central division algebra $D$ over $F$ whose degree is not divisible by $2^{2^{n-2}}$, the $K$-algebra $D \otimes_{F} K$ is a division algebra.

Moreover, the field $K$ may be chosen of the same cardinality as $F$.

Proof. If $F$ is a finite field then, since $\pi$ is anisotropic, we must have $n=1$, whence $\pi$ is the supreme form of $F$; we may then choose $K:=F$. Now we suppose that $F$ is infinite. Let $\mathcal{C}$ be the class of field extensions $K$ of $F$ such that $\pi_{K}$ is anisotropic over $K$ and such that $(i i)$ and (iii) are satisfied. This class is admissible. By (6.1), we may choose a field extension $K$ of $F$ which is of the same cardinality as $F$ and such that $K \in \mathcal{C}$ but $K(\varphi) \notin \mathcal{C}$ for any anisotropic form $\varphi$ of dimension at least 2 over $K$. Let now $\varphi$ be an anisotropic form over $K$ of dimension at least $2^{n-1}+1$. 
By Hoffmann's theorem [7, Theorem 1], every anisotropic form over $K$ of dimension not greater than $2^{n-1}$ stays anisotropic over $K(\varphi)$. Therefore $K(\varphi)$ also satisfies condition $(i i)$.

Let $C_{0}(\varphi)$ be the even part of the Clifford algebra of $\varphi$ over $K . C_{0}(\varphi)$ is a $K$-algebra of dimension $2^{\operatorname{dim}(\varphi)-1} \geq 2^{2^{n-1}}$. Let $E$ be a central division algebra over $K$ such that $E \otimes_{K} K(\varphi)$ is not a division algebra. By Merkurjev's index reduction criterion [21, Théorème 1], $E$ contains a homomorphic image of the $K$-algebra $C_{0}(\varphi)$. Hence the degree of $E$ is divisible by $2^{2^{n-2}}$. This shows that condition $($ iii $)$ is equally satisfied for the field $K(\varphi)$.

However, $K(\varphi) \notin \mathcal{C}$ by the choice of $K$, so we conclude that $\pi_{K(\varphi)}$ is isotropic. Hence $\varphi$ is similar to a subform of $\pi_{K}$ by [19, Theorem 4.5.4.(ii)], because $\pi_{K}$ is an anisotropic Pfister form. As this holds for every anisotropic form $\varphi$ over $K$ of dimension at least $2^{n-1}+1$, (3.6) shows that $\pi_{K}$ is supreme.

6.3 Proposition. Let $n \geq 2$ and let $P$ be a set of anisotropic $n$-fold Pfister forms over $F$. There exists a field extension $K / F$ such that

(i) $\pi_{K}$ is anisotropic for every $\pi \in P$,

(ii) every anisotropic form over $K$ is a subform of $\pi_{K}$ for some $\pi \in P$,

(iii) $K$ is 2-maximal with respect to every anisotropic $n$-fold Pfister form,

(iv) $I^{n+1} K=0$.

Proof. The class of field extensions $K / F$ such that over $K$ all $\pi_{K}(\pi \in P)$ are anisotropic, is admissible. By (6.1), we may choose a field extension $K / F$ in this class such that for every anisotropic form $\varphi$ over $K$ of dimension at least 2 there is some $\pi \in P$ for which $\pi_{K(\varphi)}$ is hyperbolic, whence $\varphi$ is similar to a subform of $\pi_{K}$ by [19, Theorem 4.5.4.(ii)]. Thus forms over $F$ of dimension greater than $2^{n}$ are isotropic. In particular, any $n$-fold Pfister form over $K$ is universal. It follows by (2.1) from above that every anisotropic form over $K$ is itself a subform of some form $\pi_{K}$, with $\pi \in P$. Further, any $(n+1)$-fold Pfister form over $K$ is hyperbolic, i.e. $I^{n+1} K=0$.

Let now $\psi$ be an anisotropic $n$-fold Pfister form and let $\psi^{\prime}$ denote its pure subform. For $a \in F^{\times} \backslash-F^{\times^{2}}$, the form $\psi^{\prime} \perp\langle-a\rangle$ over $K$ cannot be a subform of any $n$-fold Pfister form, hence it is isotropic, i.e. $\psi^{\prime}$ represents $a$. We conclude that $D_{K}\left(\psi^{\prime}\right)=F^{\times} \backslash-K^{\times 2}$, which means that $K$ is 2-maximal with respect to $\psi(4.4)$.

6.4 Remark. Note that, in order to be sure that the forms $\left(\pi_{i}\right)_{K}$ are all distinct, one should apply the last proposition to a set $P$ such that the 
classes in $\bar{I}^{n} F$ represented by the $n$-fold Pfister forms $\pi \in P$ and by the hyperbolic form $2^{n-1} \times\langle 1,-1\rangle$ form a subgroup.

6.5 Proposition. Let $\pi$ be an anisotropic $n$-fold Pfister form over $F$, where $n \geq 3$. Let $u$ denote either an even number greater than or equal to $2^{n}$ or the symbol $\infty$. There exists a field extension $K / F$ such that $\pi_{K}$ is the unique anisotropic $n$-fold Pfister form over $K$ and $K$ is nonreal with $u(K)=u$.

Proof. Let $F^{\prime}$ denote a function field in infinitely many variables over $F$. Note that $\pi_{F^{\prime}}$ is anisotropic and that for any $m \geq 1$ there exists a division algebra $D_{m}$ over $F^{\prime}$ which is a tensor product of $m$ quaternion algebras over $F^{\prime}$. Further, for $m \geq 1$ there exists an anisotropic quadratic form $\varphi_{m}$ over $F^{\prime}$ of dimension $2 m+2$ and of trivial discriminant, such that the Clifford algebra $C\left(\varphi_{m}\right)$ is Brauer equivalent to $D_{m}$. Since $\varphi_{m}$ is of the least possible dimension under these conditions, whenever $L / F^{\prime}$ is a field extension such that $\left(\varphi_{m}\right)_{L}$ is isotropic, $\left(D_{m}\right)_{L}$ will not be a division algebra over $L$.

Suppose now that $u=2 m+2$ for some $m \geq 2^{n-1}-1$. We consider the class of field extensions $K / F^{\prime}$ such that $\pi_{K}$ is anisotropic and such that $\left(D_{m}\right)_{K}$ is a division algebra over $K$. This class is admissible. Hence by (6.1) there exists a field extension $K / F^{\prime}$ in this class such that for any anisotropic form $\psi$ over $K$ of dimension at least two, $\pi_{K(\psi)}$ is hyperbolic or $\left(D_{m}\right)_{K}$ is not a division algebra.

Let $\psi$ be an anisotropic form in $I^{3} F$, hence with trivial Clifford invariant. By Merkurjev's index reduction criterion [21, Théorème 1], $\left(D_{m}\right)_{K(\psi)}$ is a division algebra. Therefore $\pi_{K(\psi)}$ is hyperbolic, whence $\psi$ is similar to a subform of $\pi_{K}$. This shows in particular that $\pi_{K}$ is the unique anisotropic $n$-fold Pfister form over $K$ and that $I^{n+1} K=0$, whence $K$ is nonreal.

Since $\left(D_{m}\right)_{K}$ is a division algebra, $\varphi_{m}$ stays anisotropic over $K$. Therefore $u(K) \geq \operatorname{dim}(\varphi)=2 m+2$. On the other hand, for any form $\psi$ over $K$ with $\operatorname{dim}(\psi) \geq 2 m+2 \geq 2^{n}, \pi_{K(\psi)}$ is anisotropic and by [21, Théorème 1], $\left(D_{m}\right)_{K(\psi)}$ is a division algebra, so the choice of $K$ implies that $\psi$ is isotropic. This shows that $u(K)=2 m+2=u$.

In the remaining case where $u=\infty$, the same method works if we consider instead the admissible class consisting of all field extensions $K / F^{\prime}$ such that $\pi_{K}$ is anisotropic and $\left(D_{m}\right)_{K}$ is a division algebra for every $m \geq 1$. The above arguments show that a field $K$ in this class chosen by (6.1) will be such that $\pi_{K}$ is the unique anisotropic $n$-fold Pfister form over $K$, that $K$ is nonreal with $I^{n+1} K=0$ and that all the forms $\varphi_{m}$ for $m \geq 1$ stay anisotropic over $K$, whence $u(K)=\infty$.

6.6 Corollary. For any $n \geq 3$ the class $\mathcal{H}(n)$ contains nonreal fields of $u$-invariant equal to $\infty$ or to any even number greater than or equal to $2^{n}$. $\square$ 
For $n=3$ the last result is due to Meurer [14], who further proved that the $u$-invariant of a field in $\mathcal{H}(3)$ cannot be odd. Hence (6.6) gives all the possible values for the $u$-invariant of a nonreal field in $\mathcal{H}(3)$.

6.7 Proposition. Let $\pi$ be an anisotropic 2-fold Pfister form and $\alpha$ an anisotropic Albert form over $F$. There exists a field extension $K / F$ such that $\pi_{K}$ and $\alpha_{K}$ are anisotropic and such that every anisotropic form over $K$ is similar to a subform of $\pi_{K}$ or of $\alpha_{K}$.

Proof. The class of field extensions $K / F$ such that $\pi$ and $\alpha$ stay anisotropic over $K$ is admissible. By (6.1) there exists a field extension $K / F$ such that $\pi_{K}$ and $\alpha_{K}$ are anisotropic while for any anisotropic form $\varphi$ over $K$ with $\operatorname{dim}(\varphi) \leq 2$ at least one of $\pi_{K(\varphi)}$ and $\alpha_{K(\varphi)}$ is isotropic. If $\pi_{K(\varphi)}$ is isotropic then we know that $\varphi$ is similar to a subform of $\pi_{K}$. On the other hand, if $\alpha_{K(\varphi)}$ is isotropic then a theorem of D. Leep, published in [11, Theorem 1.1], tells us that $\varphi$ is similar either to a 2-fold Pfister form or to a subform of $\alpha_{K}$. Suppose now that $\varphi$ is similar to a 2-fold Pfister form. Let $\psi$ denote the anisotropic part of $\pi_{K} \perp-\varphi$. Since $\psi$ is congruent modulo $I^{3} K$ to an anisotropic 2-fold Pfister form, it cannot be similar to a subform of an anisotropic Albert form. Hence $\psi$ is similar to a subform of $\pi_{K}$ or to a 2-fold Pfister form. Each implies that $\varphi \perp \psi$ is equal to $\pi_{K}$.

This proves that every anisotropic form over $K$ is similar to a subform of $\alpha_{K}$ or of $\pi_{K}$.

6.8 Corollary. $\mathcal{S}(3)$ is a proper subclass of $\mathcal{D}(3)$.

Proof. By (5.4) $\mathcal{S}(3)$ is contained in $\mathcal{D}(3)$. Using (6.7) one can construct a field $F$ satisfying the hypothesis of (5.12), hence such that $F \in \mathcal{D}(3) \backslash$ $\mathcal{S}(3)$.

6.9 Remark. As a consequence of (6.8) we obtain that $\mathcal{D}(n) \not \subset \mathcal{S}(n)$ for any $n \geq 3$. Indeed, given $F \in \mathcal{D}(3) \backslash \mathcal{S}(3)$ the iterated power series field $F\left(\left(X_{1}\right)\right) \ldots\left(\left(X_{n-3}\right)\right)$ belongs to $\mathcal{D}(n) \backslash \mathcal{S}(n)$, by (3.7) and (5.11).

In view of (6.6) and (6.8) we ask the following:

6.10 Question. Is there a field $F \in \mathcal{D}(3)$ with $u(F)>8$ ?

\section{Symbol length of a field with supreme form}

Let $\operatorname{Br}(F)$ denote the Brauer group of $F$ and $\mathrm{Br}_{2}(F)$ the subgroup of $\operatorname{Br}(F)$ consisting of the elements of order at most 2. The elements of $\operatorname{Br}_{2}(F)$ are the classes (modulo Brauer equivalence) of central simple algebras $A$ over $F$ 
for which $A \otimes_{F} A$ is isomorphic to a matrix algebra over $F$. By Merkurjev's theorem [13] every class in $\operatorname{Br}_{2}(F)$ is represented by a tensor product of quaternion algebras over $F$. If $A$ is a central simple $F$-algebra such that $[A] \in \operatorname{Br}_{2}(F)$ then we denote by $\ell(A)$ the least integer $m \geq 0$ such that $[A]$ is represented by a product of $m$ quaternion algebras over $F$.

The invariant $\lambda_{2}(F)$ is defined by

$$
\lambda_{2}(F):=\sup \left\{\ell(A) \mid[A] \in \mathrm{Br}_{2}(F)\right\}
$$

and called the 2-symbol length of $F$. This invariant takes its values in the set $\mathbb{N} \cup\{0, \infty\}$. Note that $\lambda_{2}(F)$ is zero if and only if every $F$-quaternion algebra is split, if and only if $I^{2} F=0$. Further $\lambda_{2}(F) \leq 1$ is equivalent to the condition that the classes in $\operatorname{Br}(F)$ which are represented by quaternion algebras form a subgroup of $\operatorname{Br}(F)$. Fields with this property have been studied in [4]. For definition and study of the $p$-symbol length $\lambda_{p}(F)$ for any prime $p$ we refer the reader to [8] and to [3]. Further information on $\lambda_{2}(F)$ can be found in [16].

Suppose that the field $F$ is nonreal. B. Kahn showed in [8] that $I^{m} F$ vanishes for $m \geq 2 \lambda_{2}(F)+3$, and also for $m=2 \lambda_{2}(F)+2$ if $-1 \in F^{\times^{2}}$. So, if $I^{n} F \neq 0$ then $\lambda_{2}(F) \geq\left[\frac{n-1}{2}\right]$. This applies in particular if $F \in \mathcal{S}(n)$. It is an open problem whether $\lambda_{2}(F)=\frac{n-1}{2}-1$ is possible when $I^{n} F \neq 0$. However, examples where $\lambda_{2}(F)=\left[\frac{n}{2}\right]$ and $I^{n} F \neq 0$ are easy to obtain for any $n \geq 1$; one may actually find such $F \in \mathcal{S}(n)$.

7.1 Example. Let $n \geq 1$. As already mentioned in (3.1), the field $F:=$ $\mathbb{C}\left(\left(X_{1}\right)\right) \ldots\left(\left(X_{n}\right)\right)$ has a supreme $n$-fold Pfister form. In particular, $I^{n} F$ is nontrivial while $I^{n+1} F=0(3.5, b)$. On the other hand, it is known that $\lambda_{2}(F)=\left[\frac{n}{2}\right]$ (cf. [8] or [3]).

If $F \in \mathcal{S}(1)$, then obviously $\lambda_{2}(F)=0$, and it is also clear that for $F \in \mathcal{S}(2)$ one has $\lambda_{2}(F)=1$. Now we give for $F \in \mathcal{S}(n), n \geq 3$, an upper bound on $\lambda_{2}(F)$ and show then that it is best possible.

7.2 Theorem. Let $n \geq 3$. If $F \in \mathcal{S}(n)$, then $\lambda_{2}(F) \leq 2^{n-2}-1$.

Proof. Let $A$ be a central simple $F$-algebra of exponent at most 2. It follows from Merkurjev's theorem [13] that $A$ is Brauer equivalent to the Clifford algebra $C(\varphi)$ of some anisotropic form $\varphi \in I^{2} F$. Now, $C(\varphi)$ is known to be a product of $\frac{\operatorname{dim}(\varphi)}{2}$ quaternion algebras where at least one of them is split. Therefore we have $\ell(A) \leq \frac{\operatorname{dim}(\varphi)}{2}-1$.

Suppose now that $F$ has a supreme $n$-fold Pfister form $\pi$. We decompose $\pi$ into $\varphi \perp \psi$ for a quadratic form $\psi$ over $F$ (3.2). Since $\pi \in I^{3} F$, the form $\psi$ 
lies in $I^{2} F$ and its Clifford algebra $C(\psi)$ is Brauer equivalent to $C(\varphi)$, hence also to $A$. By the same argument as above we obtain $\ell(A) \leq \frac{\operatorname{dim}(\psi)}{2}-1$. But since $\operatorname{dim}(\varphi)+\operatorname{dim}(\psi)=2^{n}$, we have either $\operatorname{dim}(\varphi) \leq 2^{n-1}$ or $\operatorname{dim}(\psi)<2^{n-1}$, hence in any case $\ell(A) \leq 2^{n-2}-1$.

7.3 Corollary. If $F \in \mathcal{S}(3)$, then $\lambda_{2}(F)=1$.

Proof. If $F \in \mathcal{S}(3)$, then $\lambda_{2}(F) \leq 1$ by $(7.2)$ and $\lambda_{2}(F) \neq 0$.

7.4 Corollary. Let $n \geq 0$ and let $\pi$ be an anisotropic $n$-fold Pfister form over $F$. There exists a field extension $K / F$ such that $\pi_{K}$ is supreme and $\lambda_{2}(K)=2^{n-2}-1$.

Proof. Since we can replace $F$ by a suitable purely transcendental extension of $F$ if necessary, we may assume that there exists a central division algebra $D$ over $F$ which is a tensor product of $2^{n-2}-1$ quaternion algebras. Now, using (6.2) we choose an extension $K / F$ such that $\pi_{K}$ is supreme over $K$ and $D_{K}$ is still a division algebra. Then $D_{K}$ cannot be Brauer equivalent to a product of less than $2^{n-2}-1$ quaternion algebras over $K$, so $\ell\left(D_{K}\right)=2^{n-2}-1$. This shows that $\lambda_{2}(K) \geq 2^{n-2}-1$ and the last theorem gives the opposite inequality.

\section{The level form as supreme form}

We now apply the results of the previous sections to the particular Pfister form $2^{n} \times\langle 1\rangle$ and study in particular the case where this form is supreme.

If we know that $F$ has a supreme $n$-fold Pfister form then we can read from the level $s(F)$ whether the supreme form is equal to $2^{n} \times\langle 1\rangle$.

8.1 Proposition (Hoffmann). Let $F \in \mathcal{S}(n)$ with $n \geq 1$. Then $s(F) \leq$ $2^{n}$ and the following are equivalent:

(i) $s(F)=2^{n}$,

(ii) the supreme form of $F$ is $2^{n} \times\langle 1\rangle$,

(iii) every nonzero element of $F$ is a sum of $s(F)$ squares in $F$.

Proof. Note that the level form of $F$ is anisotropic and has dimension $s(F)$ and that $u(F)=2^{n}\left(3.5\right.$, a). Therefore $s(F) \leq 2^{n}$.

( $i \Rightarrow$ iii) As $u(F)=2^{n}$ the form $2^{n} \times\langle 1\rangle$ is universal over $F$, i.e. every element of $F^{\times}$is a sum of $2^{n}$ squares in $F$.

$($ iii $\Rightarrow$ ii $)$ The supreme form of $F$ has dimension $2^{n}$ and by (3.3) it is the unique universal anisotropic form over $F$. Condition (iii) means that 
the level form of $F$ is universal, and it is anisotropic by definition. Therefore (iii) implies (ii).

$(i i \Rightarrow i)$ This is obvious.

We say that the field $F$ is level-s supreme if the form $s \times\langle 1\rangle$ is supreme over $F$. Then $s=s(F)$, in particular $s$ is a power of 2 .

Let $a \in F$. We write $\ell_{F}(a)$ for the least positive integer $l$ such that $a$ is a sum of $l$ nonzero squares over $F$, provided such an integer exists; otherwise, if $a$ is not a sum of nonzero squares in $F$, we set $\ell_{F}(a):=\infty$. In particular, for any field $F$ one has $\ell_{F}(-1)=s(F)$ and $\ell_{F}(0)=s(F)+1$. Further, for any $a \in F$, the inequality $\ell_{F}(a)+\ell_{F}(-a) \geq s(F)+1$ holds.

8.2 Proposition. Suppose that $F$ is level-s supreme. Then for every $a \in F^{\times}$ one has $\ell_{F}(a)+\ell_{F}(-a)=s+1$.

Proof. Let $m:=\ell_{F}(a)-1$. The form $m \times\langle 1\rangle \perp\langle-a\rangle$ over $F$ is anisotropic and therefore a subform of the supreme form $s \times\langle 1\rangle$. Witt cancellation shows that $-a$ is represented by $(s-m) \times\langle 1\rangle$, thus $\ell_{F}(-a) \leq s-m$. This yields $\ell_{F}(a)+\ell_{F}(-a) \leq s+1$, and the converse inequality holds in general.

8.3 Question. Is there a field $F$ which is not level-4 supreme and such that $\ell_{F}(a)+\ell_{F}(-a)=5$ holds for all $a \in F^{\times}$?

For any $m \geq 1$, we denote by $D_{F}(m)$ the set of nonzero elements of $F$ which can be written as a sum of $m$ squares. This is actually an abbreviation for $D_{F}(\varphi)$ where $\varphi$ is the form $m \times\langle 1\rangle$. If $m$ is a power of 2 then $m \times\langle 1\rangle$ is a Pfister form and as a consequence $D_{F}(m)$ is a subgroup of $F^{\times}$.

8.4 Corollary. If $F$ is level-2 $2^{n}$ supreme, then

$$
F^{\times}=D_{F}\left(2^{n-1}\right) \cup-D_{F}\left(2^{n-1}\right) .
$$

Proof. For any $a \in F^{\times}$, we have $\ell_{F}(a)+\ell_{F}(-a)=2^{n}+1$ by (8.2), thus either $\ell_{F}(a) \leq 2^{n-1}$ or $\ell_{F}(-a) \leq 2^{n-1}$.

If the field $F$ is level- $2^{n}$ supreme, then it is 2 -maximal with respect to $2^{n} \times\langle 1\rangle$ by (4.3). To see this one could also apply (8.2) to verify condition (iii) in the next proposition.

8.5 Proposition. Suppose that the field $F$ is nonreal. The following are equivalent:

(i) $F$ is 2-maximal with respect to the form $2^{n} \times\langle 1\rangle$, 
(ii) $s(F)=2^{n}$ and $s(K)<2^{n}$ for every quadratic extension $K / F$,

(iii) $D_{F}\left(2^{n}-1\right)=F^{\times} \backslash-F^{\times^{2}}$.

Furthermore, if these conditions are satisfied, then every nonzero element of $F$ is a sum of $2^{n}$ squares.

Proof. Note first that any of the conditions implies the anisotropy of $2^{n} \times\langle 1\rangle$ over $F$. Since this is a Pfister form with pure subform $\left(2^{n}-1\right) \times\langle 1\rangle$, we obtain the equivalence of $(i)$ and (iii) from (4.4). Further, the equivalence of $(i)$ and (ii) follows from (4.2). Finally, $(i i)$ and (iii) together yield $D_{F}\left(2^{n}\right)=F^{\times}$, which means that every nonzero element of $F$ is a sum of $2^{n}$ squares in $F$.

For $n=2$ the last result was obtained by Mináč and Smith [15, Theorem 4.5]. It seems to be known in general, as well as part $(a)$ of the next proposition. Indeed, combining both statements we retrieve the construction of a field of level and Pythagoras number both equal to $2^{n}$ which is outlined in the proof of [18, Proposition 7.1.5.(b)].

8.6 Proposition. Suppose that $s(F) \geq 2^{n}$.

(a) If $F$ is nonreal, then there exists a 2-extension $K / F$ such that $K$ has level $2^{n}$ and is 2-maximal with respect to the level form $2^{n} \times\langle 1\rangle$.

(b) There exists a field extension $K / F$ such that $K$ is level- $2^{n}$ supreme and $\lambda_{2}(K)=2^{n-2}-1$.

Proof. Part (a) follows from (4.1) and part (b) from (7.4), each time applied to the Pfister form $2^{n} \times\langle 1\rangle$.

8.7 Question. Given $n \geq 4$, does there exist a field $F$ which is level- ${ }^{n}$ supreme and such that $\lambda_{2}(F)<2^{n-2}-1$ ?

The following result was announced by the author in [1, Section 3].

8.8 Corollary. If $s(F)=2^{n}$ then $\left|D_{F}\left(2^{n}\right) / D_{F}\left(2^{n-1}\right)\right| \geq 2$ and this estimate is best possible for any $n \geq 1$.

Proof. If $s(F)=2^{n}$ then -1 represents a nontrivial class in the quotient $D_{F}\left(2^{n}\right) / D_{F}\left(2^{n-1}\right)$, so the estimate is clear. On the other hand, if $F$ is level- $2^{n}$ supreme then we conclude from (8.4) that $\left|D_{F}\left(2^{n}\right) / D_{F}\left(2^{n-1}\right)\right|=2$. Therefore, for any $n \geq 1$ the estimate is optimal, since level- $2^{n}$ supreme fields do exist by $(8.6, b)$. 
By (8.4), if $F$ is a level- $2^{n}$ supreme, then $D_{F}\left(2^{n-1}\right)$ is of index 2 in $F^{\times}$. Under the weaker condition that $F$ have level $2^{n}$ and be 2-maximal with respect to the level form, the quotient $F^{\times} / D_{F}\left(2^{n-1}\right)$ does not even need to be finite.

8.9 Example. Let $F$ be a field with $s(F) \geq 2^{n}$, where $n \geq 2$, such that $F^{\times} / D_{F}\left(2^{n-1}\right)$ is infinite (e.g. a real field with infinitely many orderings). Let $P$ denote the set of anisotropic $n$-fold Pfister forms of the shape $2^{n-1} \times\langle\langle-a\rangle\rangle$ for $a \in F^{\times} \backslash D_{F}\left(2^{n-1}\right)$. By (6.3), there exists a field extension $K / F$ such $\pi_{K}$ is anisotropic for any $\pi \in P, I^{n+1} K$ vanishes and $K$ is 2-maximal with respect to every anisotropic $n$-fold Pfister form. In particular, $K$ is nonreal and 2-maximal with respect to $2^{n} \times\langle 1\rangle$. As a consequence, we obtain $s(K)=2^{n}$ and $K^{\times}=D_{K}\left(2^{n}\right)$. Further, since $2^{n-1} \times\langle\langle-a\rangle\rangle$ stays anisotropic over $K$ for any $a \in F^{\times} \backslash D_{F}\left(2^{n-1}\right)$, we have $F^{\times} \cap D_{K}\left(2^{n-1}\right)=D_{F}\left(2^{n-1}\right)$. Hence $F^{\times} / D_{F}\left(2^{n-1}\right)$ injects into $D_{K}\left(2^{n}\right) / D_{K}\left(2^{n-1}\right)$, which therefore is infinite.

We have seen that the property for $F$ to be level- $2^{n}$ supreme has many consequences. However, it does not determine the Witt ring of $F$ when $n \geq 2$. In fact, the square class number of a level-4 supreme field can be finite or infinite, as the following shows.

8.10 Example. Let $K$ be an extension of odd degree $2 m+1$ of the field of dyadic numbers $\mathbb{Q}_{2}$. Then $\left|K^{\times} / K^{\times 2}\right|=2^{2 m+3}$ [12, Ch. VI, Corollary 2.23]. Then $K$ is a local field and, by Springer's Theorem, $\langle\langle 1,1\rangle\rangle$ is anisotropic over $K$. By (5.8) we conclude that $K$ is a supreme level-4 field.

Let further $L$ denote the direct limit of the finite odd degree extensions of $\mathbb{Q}_{2}$. One easily sees from the previous that $L$ is a supreme level- 4 field and that $L^{\times} / L^{\times 2}$ is infinite.

In particular $L$ is 2 -maximal with respect to its supreme form $\langle\langle 1,1\rangle\rangle$. If now $M$ is any field containing $L$ such that $M^{\times} / M^{\times 2}$ is finite, then $L$ is not quadratically closed in $M$, so $\langle\langle 1,1\rangle\rangle$ must be isotropic over $M$, i.e. $s(M) \leq 2$.

We recall that it is still an open question whether there exists a field $K$ such that $4<s(K)<\infty$ and such that $K^{\times} / K^{\times 2}$ is finite (cf. [1]). A natural approach to search for such a field $K$ might be to start with a field $F$ with $s(F) \geq 8$ and to try to construct $K$ as an extension of $F$. However, the last example showes that a field $F$ may have no extension $K / F$ such that $K^{\times} / K^{\times 2}$ is finite and $s(K)=s(F)$. In the author's interpretation, the mentioned approach is therefore unlikely to help solving the problem. It might be interesting, however, to search sufficient conditions on $F$ for it to have no extension $K / F$ such that $K^{\times} / K^{\times 2}$ is finite and such that $4<s(K)<\infty$ (or even $2<s(K)<\infty$ ). 


\section{Acknowledgements}

The author wishes to express his gratitude to Detlev Hoffmann, Ahmed Laghribi and Jean-Pierre Tignol for various remarks, which had an important influence on the development of this research. He also greatfully thanks Tatiana Beliaeva and Thomas Unger for many valuable comments, which helped improve the manuscript.

This research was started during the author's doctoral studies at Université de Franche-Comté (Besançon, France). It was continued and finished during two postdoctoral visits, at Université Catholique de Louvain (Louvain-la-Neuve, Belgium), and at University College Dublin (Ireland), both financed by the TMR network 'Algebraic $K$-Theory, Linear Algebraic Groups and Related Structures' (ERB FMRX CT-97-0107). The author wishes to thank these institutions for their hospitality and support.

\section{References}

[1] Becher, K. J. On the Number of Square Classes of a Field of Finite Level. Documenta Math., Extra Volume, Quadratic Forms LSU: 65-84, 2001.

[2] Becher, K. J. Le radical de Kaplansky. Publ. Math. Fac. Sci. Besançon, to appear, 2003.

[3] Becher, K. J.; Hoffmann, D. W. On symbol lengths in Milnor K-theory, preprint, 2003.

[4] Elman, R.; Lam, T. Y. Classification theorems for quadratic forms over fields. Comment. Math. Helv., 49:373-381, 1974.

[5] Elman, R.; Lam, T. Y. Quadratic forms under algebraic extensions. Math. Ann., 219(1):21-42, 1976.

[6] Fröhlich, A. Quadratic forms "à la" local theory. Proc. Cambridge Philos. Soc., 63:579-586, 1967.

[7] Hoffmann, D. W. Isotropy of quadratic forms over the function field of a quadric. Math. Z., 220(3):461-476, 1995.

[8] Kahn, B. Comparison of some field invariants. J. Algebra, 232(2):485492, 2000.

[9] Kaplansky, I. Fröhlich's local quadratic forms. J. Reine Angew. Math., 239/240:74-77, 1969. 
[10] Kozioł, K. Quadratic forms over quadratic extensions of generalized local fields. J. Algebra, 118(1):1-13, 1988.

[11] Laghribi, A. Formes quadratiques de dimension 6. Math. Nachr., 204:125-135, 1999.

[12] Lam, T. Y. The algebraic theory of quadratic forms. W. A. Benjamin, Inc., Reading, Mass., 1973. Mathematics Lecture Note Series.

[13] Merkurjev, A. S. On the norm residue symbol of degree 2. Dokl. Akad. Nauk SSSR, 261(3):542-547, 1981.

[14] Meurer, M. Über die u-Invariante von Körpern. Dissertation, Johannes Gutenberg-Universität Mainz, 1995.

[15] Mináč, J.; Smith, T. L. $W$-groups and values of binary forms. J. Pure Appl. Algebra, 87(1):61-78, 1993.

[16] Mináč, J.; Wadsworth, A. R. The $u$-invariant for algebraic extensions, $K$-theory and algebraic geometry: connections with quadratic forms and division algebras (Santa Barbara, CA, 1992), 333-358, Amer. Math. Soc., Providence, RI, 1995.

[17] Pfister, A. Zur Darstellung von -1 als Summe von Quadraten in einem Körper. J. London Math. Soc., 40:159-165, 1965.

[18] Pfister, A. Quadratic forms with applications to algebraic geometry and topology. Cambridge University Press, Cambridge, 1995.

[19] Scharlau, W. Quadratic and Hermitian forms. Springer-Verlag, Berlin, 1985.

[20] Szymiczek, K. Generalized Hilbert fields. J. Reine Angew. Math., 329:58-65, 1981.

[21] Tignol, J.-R. Réduction de l'indice d'une algèbre simple centrale sur le corps des fonctions d'une quadrique. Bull. Soc. Math. Belg. Sér. A, 42(3):735-745, 1990.

[22] Wadsworth, A. R. p-Henselian fields: $K$-theory, Galois cohomology, and graded Witt rings. Pacific J. Math., 105(2):473-496, 1983.

Karim Johannes Becher, Fachbereich Mathematik und Statistik, Fach D204, Universität Konstanz, D-78457 Konstanz, Germany; e-mail: becher@maths.ucd.ie

February 2003 